\title{
Reinventing Hydroacylation: A Redox-Neutral Synthesis of Ketones by Coupling of Alkenes and Amides
}

\author{
Authors: Jing $\mathrm{Li}^{\dagger}$, Rik Oost ${ }^{\dagger}$ and Nuno Maulide* \\ Institute of Organic Chemisty, University of Vienna, Währinger Strasse 38, 1090 Vienna, Austria \\ $\uparrow$ These authors contributed equally to this work.
}

\begin{abstract}
The direct synthesis of ketones via carbon-carbon bond formation represents one of the most important challenges in organic synthesis. Hydroacylation of alkenes offers perhaps the most efficient and atom-economical approach for the preparation of ketones employing carbonyl compounds and alkenes as feedstocks. State-of-the-art hydroacylation is typically achieved by a transition metal-catalyzed coupling of an aldehyde and an alkene but is plagued by competing decarbonylation, requiring the installation of directing groups in the aldehyde reactant. Herein, we present a new concept for the hydroacylation of alkenes employing amides in a metal-free regime, proceeding by an entirely new mechanism and offering orthogonal reactivity to the conventional, metal-catalysed alternatives.
\end{abstract}

Ketones and aldehydes are perhaps the quintessential functional groups of organic chemistry. Their unique ability to mediate $\mathrm{C}-\mathrm{C}$ bond forming reactions serving as either electrophiles (by direct nucleophilic addition to the carbonyl) or nucleophiles (by virtue of enolate or enamine formation) remains one of the cornerstones of the past 4 decades of organic synthesis. An overabundance of ketone syntheses rely on the direct, one-step 1,2-addition of organometallic reagents to suitable electrophilic carboxylic acid derivatives. ${ }^{[1,2]}$ Unfortunately, these nucleophilic substitutions can suffer from a number of limitations including overaddition, poor chemoselectivity regarding the presence of other carbonyl groups, excessive use of acylating reagents or tedious procedures. The advent of the venerable Weinreb amides and related derivatives ${ }^{[3,4]}$ offered a robust solution to the overaddition issue and triggered the development of related procedures relying on amide activation/organometallic addition. ${ }^{[5]}$ The recent breakthrough of Garg, Szostak and others on achieving ketone synthesis from activated amides via $\mathrm{Ni}$-catalyzed cross-coupling is perhaps the corollary of these developments. ${ }^{[6-8]}$ Nevertheless, both the Weinreb family of reactions and these 
elegant cross-coupling processes still rely on stoichiometric amounts of main group organometallics or organoboron reagents.

a<smiles></smiles>

b<smiles>[R][R][Ba][R]</smiles>

C<smiles>[R]C=CC(C)C(C)C</smiles><smiles>[R]C(CO)CC(=O)O</smiles>

d This work

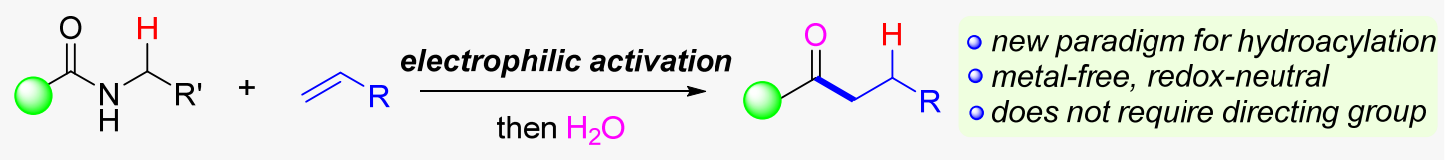

Figure 1. | Paradigms for ketone synthesis in modern organic chemistry and new hydroacylation concept using secondary amides. a, 1,2- monoaddition of organometallic reagents to Weinreb amides. b, Ni-catalyzed crosscoupling of activated amides. $\mathbf{c}$, challenges in contemporary olefin hydroacylation. $\mathbf{d}$, The present study: novel concept for intermolecular hydroacylation of olefins and amides.

Alkenes would constitute highly appealing alternatives to conventional organometallic reagents for addition to carbonyl groups en route to ketone synthesis. ${ }^{[9-11]}$ Hydroacylation of olefins has therefore emerged as a robust method for the preparation of ketones, typically achieved by the atom-economical, catalytic addition of an aldehyde $\mathrm{C}-\mathrm{H}$ bond across an alkene. ${ }^{[12,13]}$ However, contemporary transition metal-catalyzed hydroacylation presents several challenges, the most notable of which is the competing decarbonylation of the aldehyde partner prior to coupling with the alkene counterpart. Therefore, while intramolecular olefin hydroacylation has reached high levels of efficiency and selectivity, intermolecular hydroacylation still typically relies on (a) aldehydes equipped with directing groups designed to minimize decarbonylation as well as on (b) activated alkene partners. 
Aiming to address these challenges and guided by previous work on amide activation, ${ }^{[5,14-18]}$ we herein report a conceptually novel approach to olefin hydroacylation relying on an intermolecular coupling of secondary amides and alkenes, which requires neither a transition metal catalyst nor directing groups and which delivers ketones with high levels of chemo- and regioselectivity.

Table 1. Reaction discovery and optimization ${ }^{\mathrm{a}}$

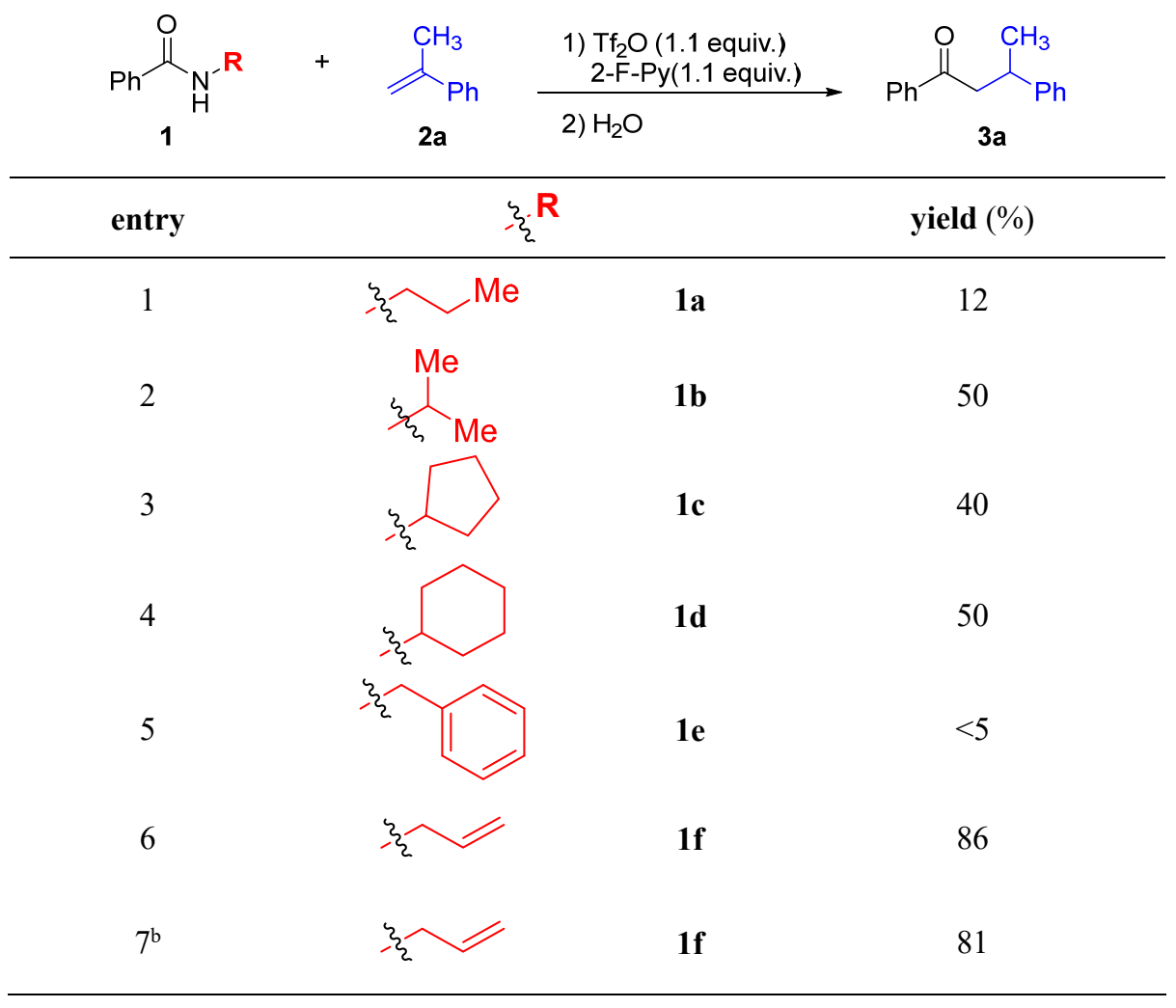

${ }^{\mathrm{a}}$ For detailed reaction conditions, see the Supplementary Information. ${ }^{\mathrm{b}} \mathrm{CH}_{3} \mathrm{CN}$ instead of $\mathrm{CH}_{2} \mathrm{Cl}_{2}$.

In initial experiments, a range of secondary amides $\mathbf{1}$ was activated prior to the addition of commercially available alkene $\mathbf{2 a}$. From the outset, trace amounts of ketone could be detected using the simple $N$-propyl amide 1a (Table 1, entry 1). Encouraged by this result, we noted that the amide $N$-substituent critically affects the efficiency of this transformation (entries 1-6). Eventually, we found that $N$-allylamides afforded high (80-90\%) and reproducible yields of the ketone 3a, a formal hydroacylation product. Additional screening of base (see Supporting Information, pages S2) and solvent effects revealed that $\mathrm{CH}_{2} \mathrm{Cl}_{2}$ and $\mathrm{CH}_{3} \mathrm{CN}$ afforded the desired ketone product in similar yields. (cf. entry 6 and entry 7) 
Table 2. Scope of the metal-free hydroacylation of alkenes.

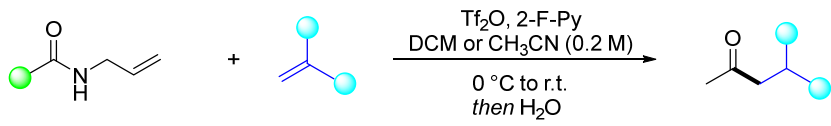

12

$3-5$

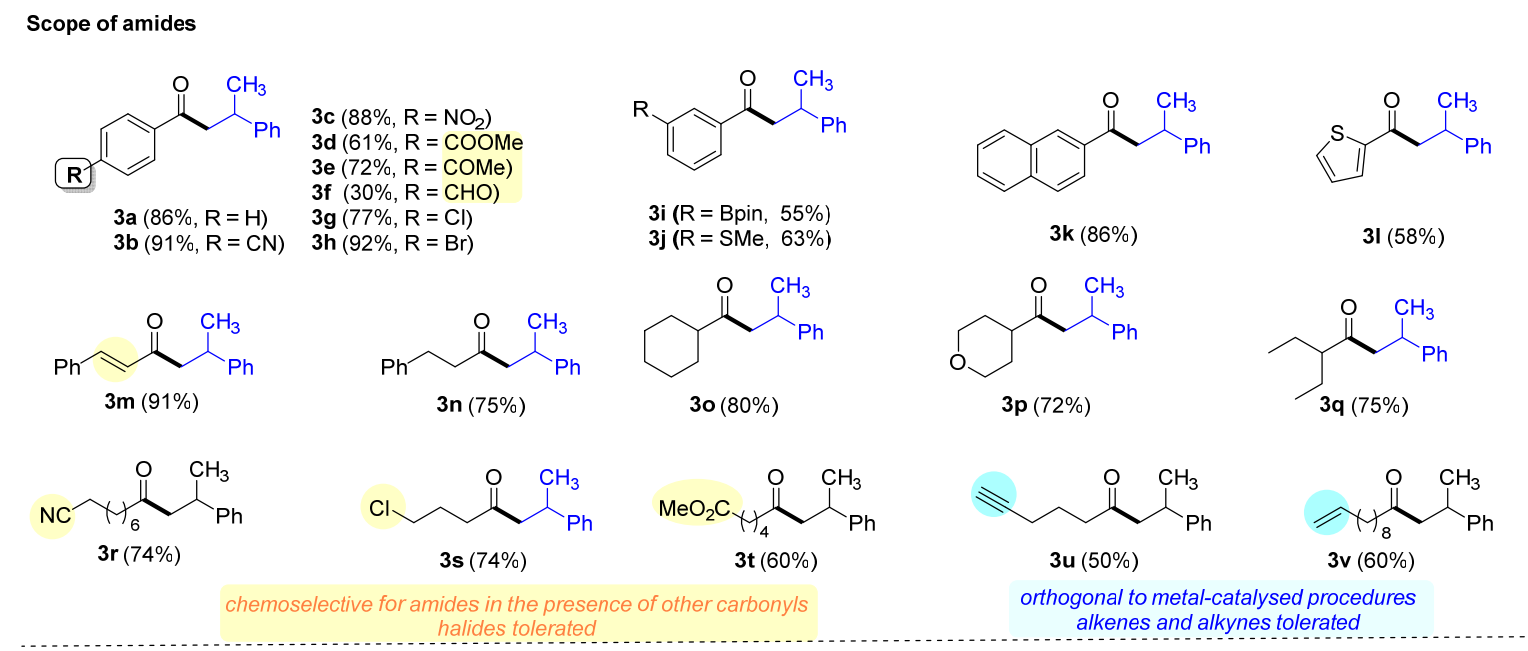

Scope of alkenes
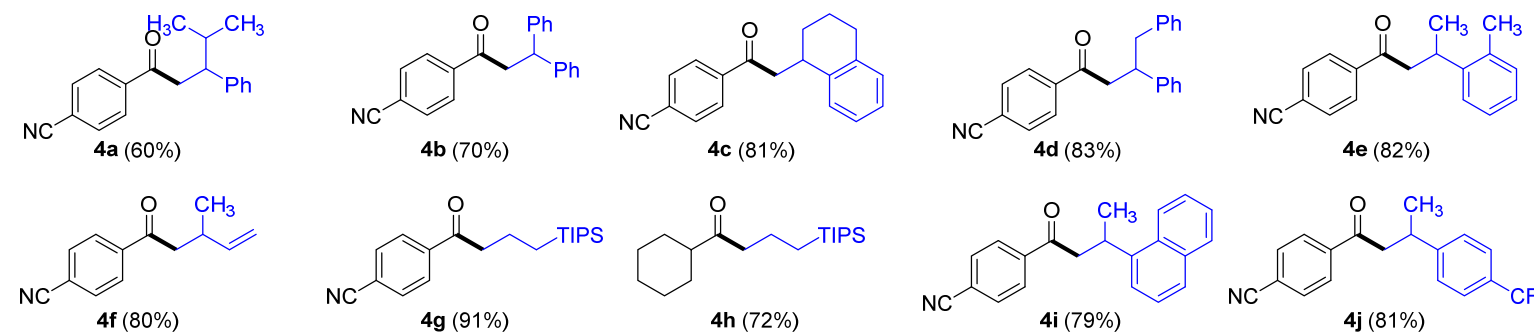

$\underbrace{O}_{4 h(72 \%)}$ TIPS


Late-stage hydroacylation of bioactive substrates
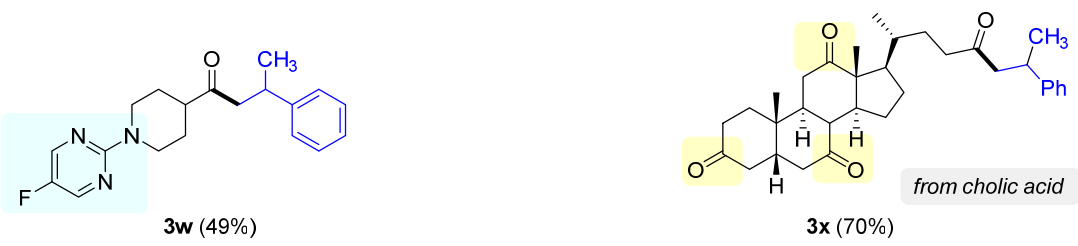

${ }^{\mathrm{a}}$ For reaction conditions and further substrates, see the Supplementary Information.

With optimized conditions in hand, we assessed the scope of the methodology. The functional group tolerance and substrate scope on the amide component of this transformation were 
investigated first. Different aromatic amides bearing valuable substituents for downstream reactions (such as $-\mathrm{CN},-\mathrm{NO}_{2},-\mathrm{COOMe},-\mathrm{Cl}$ and $-\mathrm{Br}$ ) were tolerated. Functional groups which are unlikely to tolerate organometallic reagents, such as a ketone $\mathbf{3 a}$, an aldehyde $\mathbf{3 f}$ or a boronic ester 3i also afforded the desired ketone selectively in moderate to good yields. In addition, the orthogonality of this process in respect to hydroacylation of alkenes/alkynes becomes apparent when examining aliphatic amides $\mathbf{3} \mathbf{u}-\mathbf{3 v}$, whereby alkene and alkyne moieties entailed in the amide partner behaved as spectators in this process.

At this juncture we turned our attention to the scope of alkenes. Different $\alpha$-substituted styrenes were tested and it was observed that steric hindrance doesn't affect reactivity in a pronounced manner $(\mathbf{4 a}-\mathbf{4 e})$. Electron-withdrawing substituents such as $-\mathrm{CF}_{3}$ or $-\mathrm{CN}(\mathbf{4 j}, \mathbf{4 k})$ still afforded the desired ketone in good chemical yield. More importantly, a simple 1,3-diene (4f) was also a suitable substrate for this transformation. The use of an allyl silane led to what could be termed an "interrupted allylation", delivering a product $(\mathbf{4 h})$ where silicon is retained. The methodology was also applied with success to heterocyclic and more complex amides with a drug-like framework $^{[19,20]}$ containing Lewis basic pyrimidine moieties $(\mathbf{3 w})$ or the late-stage functionalization of dehydrocholic acid (3x).

We then sought to obtain experimental information on the reaction mechanism (Figure 2). The use of deuterated amide $\mathbf{d}_{\mathbf{1}} \mathbf{- 1} \mathbf{b}$ led to selective deuterium incorporation at the carbon $\beta$-to the ketone carbonyl (Fig. 2a). This is suggestive of a hydride transfer event. On the other hand, the use of bisdeuterated $\alpha$-methylstyrene $\mathbf{d}_{\mathbf{2}}$-3a led to deuterium incorporation in the $\alpha$-position of ketone $\mathbf{3}$ (Fig. 2b). Finally, quenching the reaction with $\mathrm{H}_{2}{ }^{18} \mathrm{O}$ (20 equiv.) generated a ketone product whereby the incorporation of ${ }^{18} \mathrm{O}$ is $>90 \%$ (Fig. 2c). These observations allow us to put forth a mechanistic proposal as depicted in Fig. 2d. Thus, activation of the amide starting material is likely to generate an $N$-allyl nitrilium species $6{ }^{[21]}$ for which we also have obtained in situ NMR evidence (see Supporting Information for details, pages S23). This electrophile is captured by the alkene partner, setting up the stage for intramolecular 1,5-hydride delivery. This step simultaneously achieves reduction of the transient carbocation $\mathbf{7}$ and formation of an azoniaallene intermediate $\mathbf{8}$, the hydrolysis of which results in the hydroacylation products. ${ }^{[22]}$ 
a
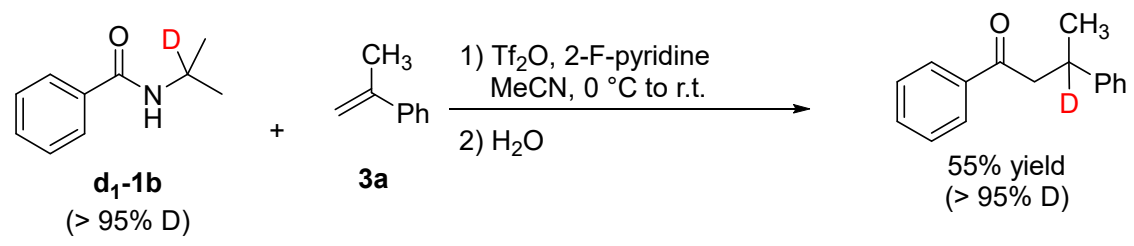

b

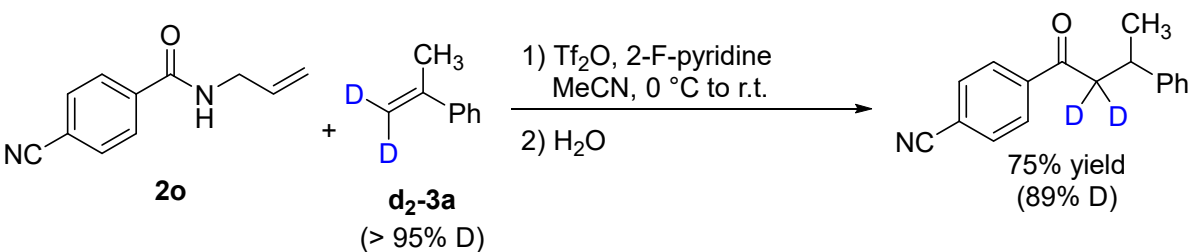

C
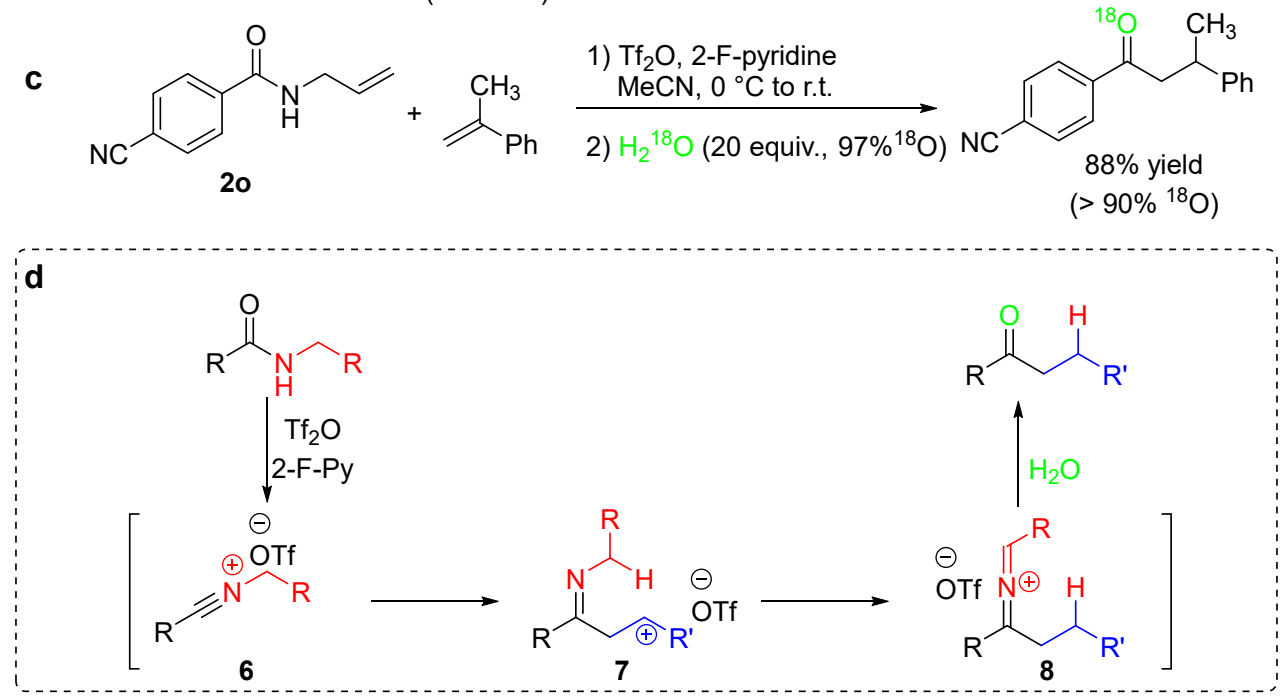

Figure 2 | Control studies and proposed mechanism.

The herein presented approach to olefin hydroacylation is a novel concept whereby ketone synthesis can be achieved by the direct, metal-free coupling of secondary amides and alkenes. This obviates the need for transition metal catalysis and proceeds by an entirely new mechanism that neither requires directing groups nor suffers from deleterious decarbonylation. This work also showcases the potential of carefully designed internal hydride transfer events to provide unique solutions for fundamental, contemporary challenges in organic synthesis.

Funding. We acknowledge funding by the Austrian Science Fund (FWF, Grant P30226) and the European Research Council (CoG 682202, VINCAT). Continued support of our research programs by the University of Vienna is gratefully acknowledged. 


\section{References and Notes:}

1. Dieter, R. K. Reaction of acyl chlorides with organometallic reagents: a banquet table of metals for ketone synthesis. Tetrahedron 55, 4177-4236 (1999).

2. Katritzky, A. R., Le, K. N. B., Khelashvili, L. \& Mohapatra, P. P. Alkyl, unsaturated, (hetero)aryl, and N protected aamino ketones by acylation of organometallic reagents. J. Org. Chem. 71, 9861-9864 (2006).

3. Balasubramaniam, S. \& Aiden, I. S. The growing synthetic utility of the Weinreb amide. Synthesis, 3707-3738 (2008).

4. Sengupta, S., Mondal, S. \& Das, D. Amino acid derived morpholine amides fornucleophilica-amino acylation reactions: a new synthetic route to enantiopurea-amino ketones. Tetrahedron Lett. 40, 4107-4110 (1999).

5. Bechara, W. S., Pelletier, G. \& Charette, A. B. Chemoselective synthesis of ketones and ketimines by addition of organometallic reagents to secondary amides. Nat. Chem. 4, 228-234 (2012).

6. Dander, J. E. \& Garg, N. K. Breaking Amides using Nickel Catalysis. ACS Catal. 7, 1413-1423 (2017).

7. Weires, N. A., Baker, E. L. \& Garg, N. K. Nickel-Catalysed Suzuki-Miyaura Coupling of Amides. Nat. Chem. 8, 7579 (2016).

8. Shi, S., Meng, G. \& Szostak, M. Synthesis of Biaryls through Nickel-Catalyzed Suzuki-Miyaura Coupling of Amides by Carbon-Nitrogen Bond Cleavage. Angew. Chem. Int. Ed. 55, 6959-6963 (2016).

9. Nguyen, K. D., Park, B. Y., Luong, T., Sato, H., Garza, V. J. \& Krische, M. J. Metal-catalyzed reductive coupling of olefin-derived nucleophiles: Reinventing carbonyl addition. Science 354, 300-306 (2016).

10. Hong, Y.-T., Barchuk, A., Krische, M. J. Branched-Selective Intermolecular Hydroacylation: Hydrogen-Mediated Coupling of Anhydrides to Styrenes and Activated Olefins. Angew. Chem. Int. Ed., 128, 6885 (2006).

11. Yujing Zhou, Jeffrey S. Bandar, and Stephen L. Buchwald,Enantioselective CuH-Catalyzed Hydroacylation Employing Unsaturated Carboxylic Acids as Aldehyde Surrogates, J. Am. Chem. Soc., 139, 8126-8129 (2017).

12. Ghosh, A., Johnson, K. F., Vickerman, K. L., Walker Jr. J. A. \& Stanley L. M. Recent advances in transition metalcatalysed hydroacylation of alkenes and alkynes. Org. Chem. Front. 3, 639-644 (2016).

13. Willis, M. C. Transition Metal Catalyzed Alkene and Alkyne Hydroacylation. Chem. Rev. 110, 725-748 (2010).

14. Kaiser, D. \& Maulide, N. Making the Least Reactive Electrophile the First in Class: Domino Electrophilic Activation of Amides. J. Org. Chem. 81, 4421-4428 (2016).

15. Movassaghi, M. \& Hill, M. D. Single-Step Synthesis of Pyrimidine Derivatives. J. Am. Chem. Soc. 128, 14254-14255 (2006).

16. Movassaghi, M. Hill, M. D. \& Ahmad, O. K. Direct Synthesis of Pyridine Derivatives, J. Am. Chem. Soc. 129, 1009610097 (2007).

17. Huang, P.-Q., Huang, Y.-H., Geng, H. \& Ye, J.-L. Metal-Free C-H Alkyliminylation and Acylation of Alkenes with Secondary Amides. Sci. Rep. 6, 28801 (2016).

18. Jochims, J. C., Hehl, S. \& Herzberger, S. Preparation and Beckman rearrangement of o-(chiorooxalyl)oximes. Synthesis 1128-1133 (1990).

19. Anderson, N. A., Bandyopadhyay, D., Daugan, A. C.-M., Donche, F. G., Eidam, P. M., Faucher, N. E., George, N. S., Harris, P. A., Jeong, J. U., King, B. W., Sehon, C. A., White, G. V \& Wisnoski, D. D. Preparation of heterocyclic amides as RIP1 kinase inhibitors for therapy. PCT Int. Appl. 177 (2016). CODEN:PIXXD2; WO2016185423.

20. Fang, J., Tang, J., Carpenter, A. J., Peckham, G., Conlee, C. R., Du, K. S. \& Katamreddy, S. R. Preparation of piperidine derivatives as GPR119 agonists for treating metabolic disorders. PCT Int. Appl. 224 (2008) CODEN:PIXXD2; WO2008070692.

21. van Dijk, T. Slootweg, J. C. \& Lammertsma, K. Nitrilium ions-synthesis and applications. Org. Biomol. Chem. 15, 10134-10144 (2017).

22. Abu-El-Halawa, R. \& Jochims, J. C. On the Reaction of N-Alkylnitrilium Salts with Acetylenes: A New Synthesis of 2-Azoniaallene Salts. Synthesis, 9, 871-874 (1992). 